\title{
Edge scaling of soap froth
}

\author{
K. Y. Szeto, and W. Y. Tam \\ Department of Physics \\ The Hong Kong University of Science and Technology \\ Clear Water Bay, Kowloon, Hong Kong. \\ Email: PHSZETO@USTHK.UST.HK
}

(April 15, 1998)

\begin{abstract}
We investigate the scaling properties of the edge distribution of soap froth and compare it with the area scaling law. The data collapse for edge scaling of cells belonging to the same topological class is explained by a harmonic expansion of the energy of the froth with area-perimeter constraint. The edge scaling hypothesis is found to be comparable to area scaling. The implication on existing theories for the evolution of soap froth is discussed.

PACS numbers:82.70Rr, 02.50.-r, 05.07.Ln
\end{abstract}

Typeset using REVTEX 
The scaling behavior of evolving soap froth has recently been the subject of many theoretical works $[1,2]$. There are two scaling behaviors that are observed in experiments. Stavans [3] has reported that not only the probability distribution $\left(P_{n}(t)\right)$ of the cells of given number of sides verses the number of edges $(n)$ is independent of time over a long period of time, but also the distribution function of area is invariant in form over the same period: $P(A, t)=f(x) / \bar{A}$ with $x=A / \bar{A}$ and the time dependence only enters in the mean area $\bar{A}(t)$. These two time invariant distribution functions $\left(P_{n}\right.$ and $\left.f(x)\right)$ define the scaling regime in the literature [4]. According to the theoretical basis of area scaling [2], one also expects that similar scaling laws apply to the subset of cells with same number of edges. However, detailed experimental test of area scaling for individual topological class (i.e. cells with same $n$ ) has not been made. In this short note, we compare the quality of data collapse for soap froth using the area scaling with edge scaling for individual topological class. We find that edge scaling is at least as good a description for experiments on soap as area scaling. Furthermore, there are physical reasons supporting the edge scaling description.

We will examine three forms of scaling function. Firstly, one uses the original area scaling hypothesis. We test area scaling of individual topological class by assuming that the probability $P_{n}^{A}\left(A_{n}, t\right)$ of finding an $n$-sided cell with area $A_{n}$ at time $t$ among all $n$-sided cells to have the following form where

$$
P_{n}^{A}\left(A_{n}, t\right)=\frac{F_{n}\left(x^{\prime}=\frac{A_{n}}{\bar{A}}\right)}{\bar{A}}, \quad \text { with } \quad \int_{0}^{\infty} d A_{n} P_{n}^{A}\left(A_{n}, t\right)=1.0
$$

Note that $P_{n}^{A}\left(A_{n}, t\right)$ is a function extracted from the histogram of area distribution of the $\mathrm{n}$-sided bubbles at time $t$ and the normalization is 1 . This form of scaling function implies that a data collapse on a universal curve of $F_{n}\left(x^{\prime}\right)=\bar{A}(t) P_{n}^{A}\left(A_{n}, t\right)$ vs $x^{\prime}=\frac{A_{n}}{A(t)}$ will be observed. The quality of the data collapse with this form is shown in Fig.1. We see that $F_{n}\left(x^{\prime}\right)$ is far from a universal curve.

Secondly, we test area scaling of a given topological class $n$ using the mean area of the $n$-sided cells. Here we assume

$$
P_{n}^{A}\left(A_{n}, t\right)=\frac{f_{n}\left(x=\frac{A_{n}}{A_{n}}\right)}{\bar{A}_{n}}, \quad \text { with } \quad \int_{0}^{\infty} d A_{n} P_{n}^{A}\left(A_{n}, t\right)=1,
$$


here $\bar{A}_{n}$ is its average area of all $n$-sided cells at time $t$. This form of scaling function implies that a data collapse on a universal curve of $f_{n}(x)=\bar{A}_{n}(t) P_{n}^{A}\left(A_{n}, t\right)$ vs $x=\frac{A_{n}}{A_{n}(t)}$ will be observed. This form differs from Eq.1 in that $\bar{A}_{n}$, the mean area of the $n$-sided cells, replaces $\bar{A}$, the mean area of all cells in the scaling variable $x$. The quality of this area scaling form is shown in Fig.2.

The third form to be tested is based on edge scaling. Here we denote the probability $P_{n}^{L}\left(L_{n}, t\right)$ of finding an $n$-sided cell with perimeter $L_{n}$ at time $t$ among all $n$-sided cells to have the following form

$$
P_{n}^{L}\left(L_{n}, t\right)=\frac{g_{n}\left(y=\frac{L_{n}}{L_{n}}\right)}{\bar{L}_{n}}, \quad \text { with } \quad \int_{0}^{\infty} d L_{n} P_{n}^{L}\left(L_{n}, t\right)=1
$$

here $\bar{L}_{n}$ is its average perimeter of all $n$-sided cells at time $t$ and $P_{n}^{L}$ is a function extracted from the histogram of edge distribution of the n-sided bubbles at time $t$. Fig.3 shows the data collapse with edge scaling.

The quality of data collapse using Eq.1 is much worse than that in Eq.2. On the other hand, the data collapse in Eq.3 is comparable, if not better than that in Eq.2. The error in the width $(W)$ of three scaling functions for $n=4,5, . ., 9$ are summarized in Table 1 . We see that the ratio of the error over mean width for Eq.2 and Eq.3 are comparable.

We show the comparison of the data collapse in Fig.1-3 for $n=5,6$, and 7. The statistics are taken from an experiment of two-dimensional soap froth where there are initially more than 20,000 bubbles and evolving to a state of 1500 bubbles over the period of more than 30 hours [5]. The data collapse for $P_{n}$ becomes time independent for $t \geq 6$ hours, corresponding to systems with less than 6000 bubbles. We have therefore analysed the data for $t \geq 6$ hours, and selected the following time values: $t=7, \ldots, 29$ hours in 2 hours interval. Since the fraction $P_{n}$ of n-sided bubbles for $n=5,6,7$ is about $0.31,0.34,0.20$, and much smaller for other $n$, we only analyse the data for these three dominant $n$. From Fig.1-3, we see that the edge scaling hypothesis Eq.3 is very good in collapsing the data.

We now present a simple model to explain the edge scaling hypothesis. In the simplest model, the energy of the froth is the sum of the energy of individual bubbles. Each bubble 
$i$ is characterized by its area $a_{i}$, perimeter $L_{i}$, topological class $n_{i}$ and the position of its center of mass $\vec{R}_{i}$. The energy of bubble $i$ is $E_{i}$ and the dominant contribution is the surface energy $\gamma L_{i}$ where $\gamma$ is the surface tension. We can then write the total energy of the froth with $N$ cells as

$$
E=\sum_{i}^{N} E_{i}=\sum_{n=3}^{\infty} \sum_{j=1}^{N_{n}} \gamma \bar{L}_{n}+\frac{1}{2} \lambda\left(L_{j}-\bar{L}_{n}\right)^{2} .
$$

Here $\bar{L}_{n}$ is the average length of perimeter of all the n-sided cells, (there are $N_{n}=N P_{n}$ of them). The grand partition function of the froth at a given time $t$ is $Z=\sum e^{E}$ where the sum indicates all configurations of these bubbles subjected to appropriate constraints to be discussed later. In particular, we can write $Z=\sum_{N=0}^{\infty} e^{\alpha N} z_{N}$ with $N=\sum_{n=3} N_{n}$ and $\alpha$ a Lagrange multiplier controlling the cell number $N$. The canonical partition function is

$$
z_{N}=\prod_{j=1}^{N} \frac{1}{N !} \int d \vec{R}_{j} \int d L_{j} \int d a_{j} e^{-\beta E}
$$

Here the prime denotes the appropriate topological constraints such as the Aboav-Weaire law $[6,7]$ on the configuration space. Since the coordinate $\vec{R}_{j}$ can be integrated out to yield the total area $A_{T}$, we can simplify $z_{N}$ as $z_{N}=\frac{A_{T}^{N} Q^{N}}{N !}$ with

$$
Q=\sum_{n=3}^{\infty} \int_{0}^{\infty} d L \int d a C_{n}(a, L) e^{-\beta \gamma \bar{L}_{n}-\frac{1}{2} \beta \lambda\left(L-\bar{L}_{n}\right)^{2}}
$$

Here the limit of integration for the area is constrained by the function $C_{n}(a, L)$ which describes the geometric relation between the perimeter and area of an n-sided bubble as well as any constraints related to the correlations in froth. Since the maximum area for an n-sided bubble with a given value of perimeter is that of a regular $n$-sided regular polygon, the constraint function $C_{n}(a, L)$ can be replaced by requiring area to assume value between zero and $A^{\star}=K_{n} L^{2}$ for the $n$-sided polygon. The value of $K_{n}$ is given in the appendix. We do not include the correlation effects here in this short paper, as correlation should only modify the parameters of the model, but not the form of the harmonic approximation that we discussed below. With these preparation, the single cell partition function $Q$ can be written as $Q=\sum_{n=3}^{\infty} \int_{0}^{\infty} d L G(L, n)$ with the edge distribution function given by 


$$
G(L, n)=e^{-\beta \gamma \bar{L}_{n}} e^{-\frac{1}{2} \beta \lambda\left(L-\bar{L}_{n}\right)^{2}} \int_{0}^{A^{\star}} d a e^{-\beta \omega a}
$$

Here $\omega$ is the energy per unit area of the bubble. This function is the origin of the scaling function for the edge. The parameter $\lambda$ is phenomenological.

Thus far we have only adopted the method of equilibrium statistical mechanics to calculate the partition function of a system which is actually evolving. In order to have scaling, meaning an edge distribution function of the form of Eq.3, we need to incorporate the evolution of the mean edge length into this equilibrium description, assuming of course that the evolution is sufficiently slow. We now use dimensional analysis to obtain the form of the scaling function $G(L, n)$ based on the harmonic description of the energy:

$$
G(L, n)=\exp \left(-\alpha_{1} \frac{L_{n}}{s_{n}(t)}-\alpha_{2}\left(\frac{L-L_{n}}{s_{n}(t)}\right)^{2}\right) \int_{0}^{A_{\star}} d a e^{-\beta \omega a} .
$$

Here $s_{n}(t)$ is the length unit at time $t$ and can be taken as the average edge length of the $n$-sided polygons. Indeed, we have $L_{n}(t)=n s_{n}(t)$. One can see immediately that if we treat the area contribution to the energy in the area integral in $G(L, n)$ as constant, then $G(L, n)$ is simply a Gaussian of the form

$$
G(L, n)=\exp \left(-\alpha_{1} n-\alpha_{2} n^{2}(y-1)^{2}\right) .
$$

The parameters $\alpha_{1}$ and $\alpha_{2}$ are phenomenological, and their determinations require a detailed model of soap froth and the constraint term in $G(L, n)$. Here the important thing is the form of $G$ in terms of the scaling variable $y$. Here $G$ has a mean at $y=L / L_{n}=1$ and a variance $\sigma^{2}$ proportional to $1 / n^{2}$. Thus a plot of the square root of $(1 / \sigma)$ versus $n$ should be linear. Fig.4 shows that this indeed is a fair comparison with our data for $g_{n}(y)$. The slope in Fig. 4 is given by $\sqrt{2 \alpha_{2}}$, yielding $\alpha_{2}=0.054 \pm 0.007$. One can improve the comparison further by a more detailed analysis of the area constraint term in the calculation of $G(L, n)$. However, using the variances of the edge scaling data from experiments, one can also plot the normalized Gaussian functions defined by these varaiances. The Gaussian functions shown in the solid lines in Fig. 3 suggest that our simple phenomenology decribes quite well the data of edge scaling. 
Now that we have a simple view of the edge scaling, which is a quasi-equilibrium description with the harmonic approximation for the independent bubble picture, we may want to question the ability of existing theories of the evolution of froth in producing edge scaling. All existing theories [2,3,10] are based on the von Neumann's law [8] and have used either the area scaling hypothesis or the scaling hypothesis of $P_{n}$ versus $n$. We think that the von Neumann's law and the very good experimental evidence of $\bar{A}$ being linear in time must be respected. The skepticism we have on existing theories is based on the fact that they either use area scaling as the starting point of calculation, or they deduce area scaling from the calculation. In terms of data collapse, we think that edge scaling is at least as good a description as area scaling, and we have presented a simple phenomenological argument for edge scaling. Now, how do existing theories accommodate the observation of edge scaling?

An important check of the edge scaling description is to see if it yields a linear time dependence of the average cell area. According to Mullins [9] and Flyvbjerg [2], the linear time dependence of the mean area is due only to the disappearance of the cells with $n=$ 3,4,5. This implies that the correct scaling must be area. However, we find here that the edge scaling is better and at the same time mean area increases with time linearly. Our conclusion is that the arguments used by Mullins and Flyvbjerg are incomplete, in that they neglect $\mathrm{T} 1$ processes which are numerous and fast processes that can change the topological class of the neighboring cells. First of all, from our studies of the dynamics of froth [11] we conclude that these $\mathrm{T} 1$ switching processes cannot be ignored. If one include T1 processes, then the derivation of the linear time dependence of the mean area is not straight forward. Consequently, we can still have edge scaling in harmony with linear time dependence of the mean area. In a different perspective, one can argue that there is no contradiction of edge scaling and area scaling at infinite time; however, in the time frame of the experiments at hand, edge scaling is archieved by the system faster, thereby giving a very good data collapse of the data. Our edge scaling hypothesis is simple and can be used to provide a phenomenological description of area scaling function, without going through the stochastic equation using von Neumann dynamics. However, we should remark here 
that any evolution theory for froth without correlation effects properly incorporated is not realistic, as experimental evidences for strong and long range correlation exist for real soap froth [12,13]. Our observation of edge scaling for individual topological class therefore poses new challenge for the theoretical understanding of evolving soap froth.

\section{Acknowledgments}

K.Y. Szeto acknowledges support from the Hong Kong Telecom Institute of Information Technology and the discussion with T. Aste, J. Stavans and especially H. Flyvbjerg. W.Y. Tam acknowledges support from the Direct Allocation Grant 96/97.SC22 of the Hong Kong University of Science and Technology.

\section{APPENDIX A: THE RELATION BETWEEN PERIMETER AND AREA OF AN N-SIDED POLYGON IN SOAP FROTH}

For a polygon with n-sides, $n>6$, the pressure inside is less than outside and the majority of edges is concave; while for $n<6$, the opposite is true with majority of the edges being convex. To calculate the area correction due to the curvature of the edges of an n-sided polygon, we make use of the condition for mechanical stability of trivalent structure, namely, that the three edges must meet at a vertex with angles equal to $2 \pi / 3$. The result of simple geometric analysis yields $A_{n}=K_{n} L_{n}^{2}$ with

$$
K_{n}=\frac{1}{4 n^{2}}\left(\frac{\sin \theta}{\theta}\right)^{2}\left[\cot \frac{\pi}{n} \pm \Delta(\theta)\right]
$$

where the plus sign holds for $n<6$ and the minus sign for $n>6$. $\Delta$ is the following function of $\theta=\pi\left|\frac{1}{n}-\frac{1}{6}\right|$

$$
\Delta(\theta)=\frac{\theta}{\sin \theta^{2}}-\cot \theta
$$




\section{REFERENCES}

[1] J. Stavans, E. Domany, and D. Mukamel, Europhys. Lett. 15, 479 (1991)

[2] H. Flyvbjerg, Phys. Rev. E, 47, 4037 (1993), Physica A194, 298 (1993)

[3] J. Stavans, and J.A. Glazier, Phys. Rev. Lett. 62, 1318 (1989)

[4] J. Stavans, Rep. Prog. Mod. Phys. 56, 733 (1993).

[5] W. Y. Tam, R. Zeitak, K. Y. Szeto, and J. Stavan, Phys. Rev. Lett. 79, 1588 (1997).

[6] D. A. Aboav, Metalloraphy 3, 383-90 (1974); 13, 43-58 (1980).

[7] D. Weaire, Metallography 7, 157-60 (1974).

[8] J. von Neumann, Metal Interfaces, (Cleveland, Ohio, American Society for Metals) 108 (1952)

[9] W.W. Mullins, Scr. Metall, 22, 1411 (1988). (Cleveland, Ohio, American Society for Metals) $108(1952)$

[10] J.R. Iglesias and Rita M.C. de Almeida, Phys. Rev. A43, 2763 (1991).

[11] K.Y. Szeto, P. Rujan, and W.Y. Tam, preprint.

[12] K.Y. Szeto and W.Y. Tam, Phys. Rev. E,53, 4213 (1996).

[13] T. Aste, K.Y. Szeto, and W.Y. Tam, Phys. Rev. E 54 (1996) 5482 -92.; K. Y. Szeto, T. Aste and W. Y. Tam, cond-mat/9707200. (1997) xxx.lanl.gov 


\section{Figures}

Fig.1 Area scaling distribution function $F_{n}\left(x^{\prime}\right)$ for soap froth at various times after 6 hours. ((a) $n=5:$ circle, (b) $n=6:$ square, (c) $n=7:$ diamond. ) Here the unit of area is mean area $\bar{A}$ for all cells.

Fig.2 Area scaling distribution function $f_{n}(x)$ for soap froth at various times after 6 hours. ((a) $n=5$ : circle, (b) $n=6:$ square, (c) $n=7:$ diamond. ) Here the unit of area is mean area $\bar{A}_{n}$ for all $n$-sided cells.

Fig. 3 Edge scaling distribution function $g_{n}(y)$ for soap froth at various times after 6 hours. ((a) $n=5:$ circle, (b) $n=6:$ square, (c) $n=7:$ diamond. ) Here the unit of edge length is mean perimeter $\bar{L}_{n}$ for all $n$-sided cells. The solid lines correspond to the theoretical curves.

Fig.4 Inverse of the standard deviation $\sigma$ of the edge distribution function for a given topological class vs $n$. 


\section{TABLES}

TABLE I. Table 1. Mean, Standard Deviation and Fractional error of width $W$ of Scaling Functions

\begin{tabular}{|c||c|c|c||c|c|c||c|c|c||}
\hline \hline $\mathrm{n}$ & $\left\langle W_{F}\right\rangle$ & $\sigma_{W_{F}}$ & $\sigma_{W_{F}} /\left\langle W_{F}\right\rangle$ & $\left\langle W_{f}\right\rangle$ & $\sigma_{W_{f}}$ & $\sigma_{W_{f}} /\left\langle W_{f}\right\rangle$ & $\left\langle W_{g}\right\rangle$ & $\sigma_{W_{g}}$ & $\sigma_{W_{g}} /\left\langle W_{g}\right\rangle$ \\
\hline \hline 4 & 0.0028 & 0.0004 & 0.128 & 0.3255 & 0.0048 & 0.015 & 0.4114 & 0.0119 & 0.029 \\
\hline 5 & 0.0023 & 0.0003 & 0.149 & 0.3209 & 0.0026 & 0.008 & 0.3492 & 0.0038 & 0.011 \\
\hline 6 & 0.0014 & 0.0002 & 0.164 & 0.2153 & 0.0037 & 0.017 & 0.2102 & 0.0031 & 0.015 \\
\hline 7 & 0.0010 & 0.0002 & 0.167 & 0.1546 & 0.0034 & 0.022 & 0.1483 & 0.0029 & 0.019 \\
\hline 8 & 0.0009 & 0.0001 & 0.165 & 0.1296 & 0.0021 & 0.017 & 0.1272 & 0.0018 & 0.015 \\
\hline 9 & 0.0007 & 0.0001 & 0.189 & 0.1037 & 0.0040 & 0.039 & 0.1014 & 0.0037 & 0.037 \\
\hline \hline
\end{tabular}


Szeto et al Fig. 1a

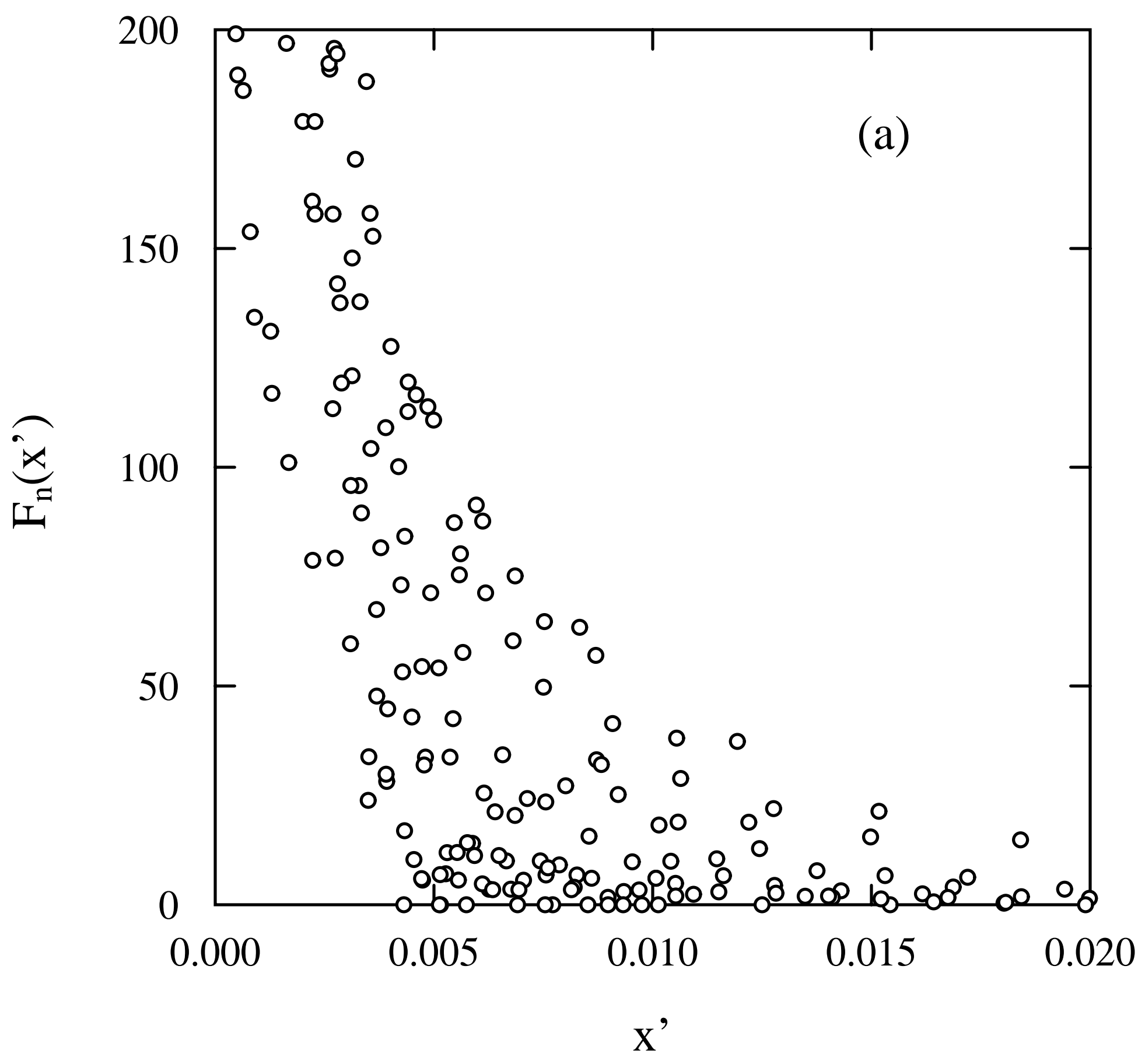


Szeto et al Fig. 1b

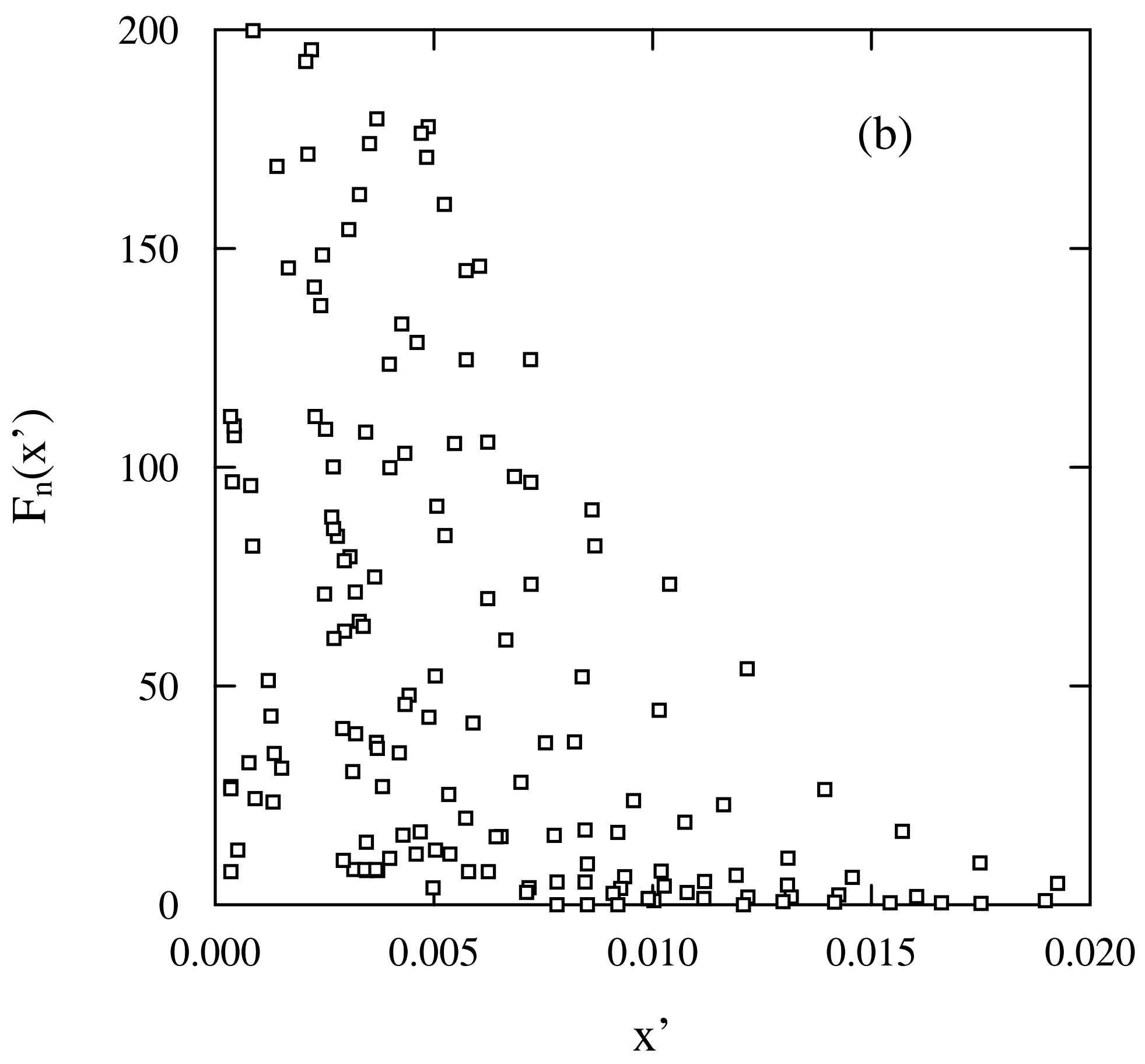


Szeto et al Fig. 1c

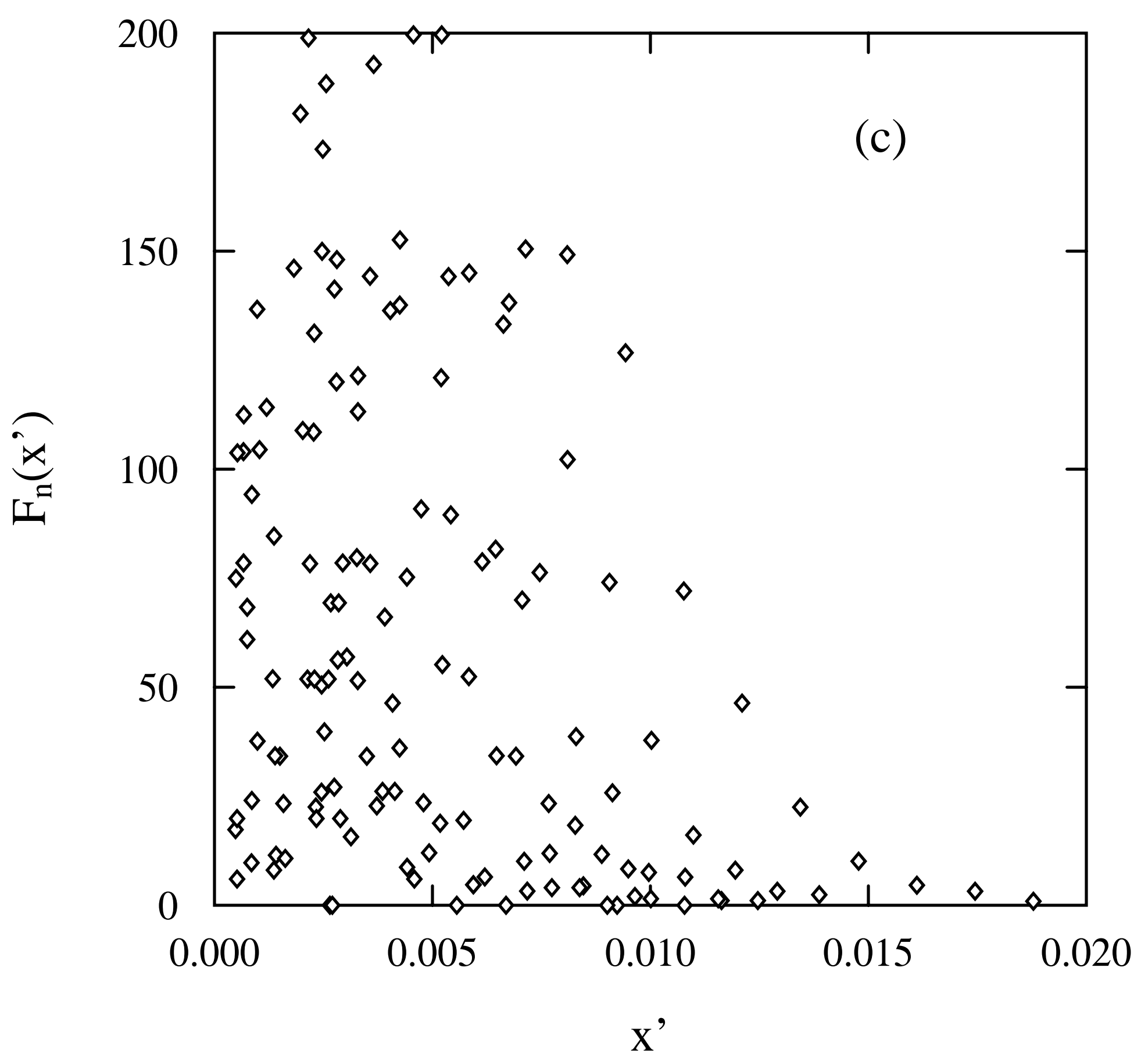


Szeto et al Fig. 2a

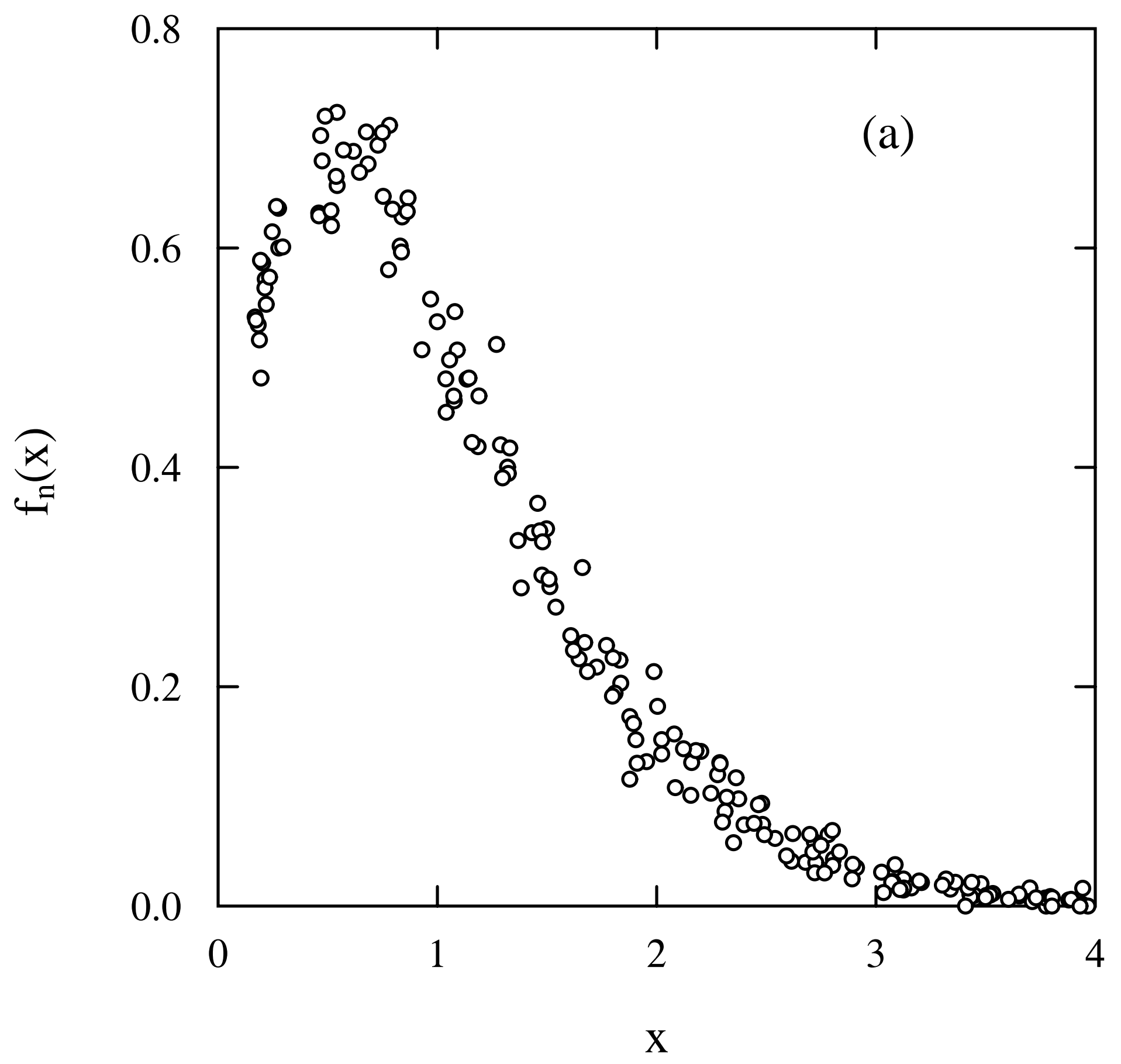


Szeto et al Fig. 2b

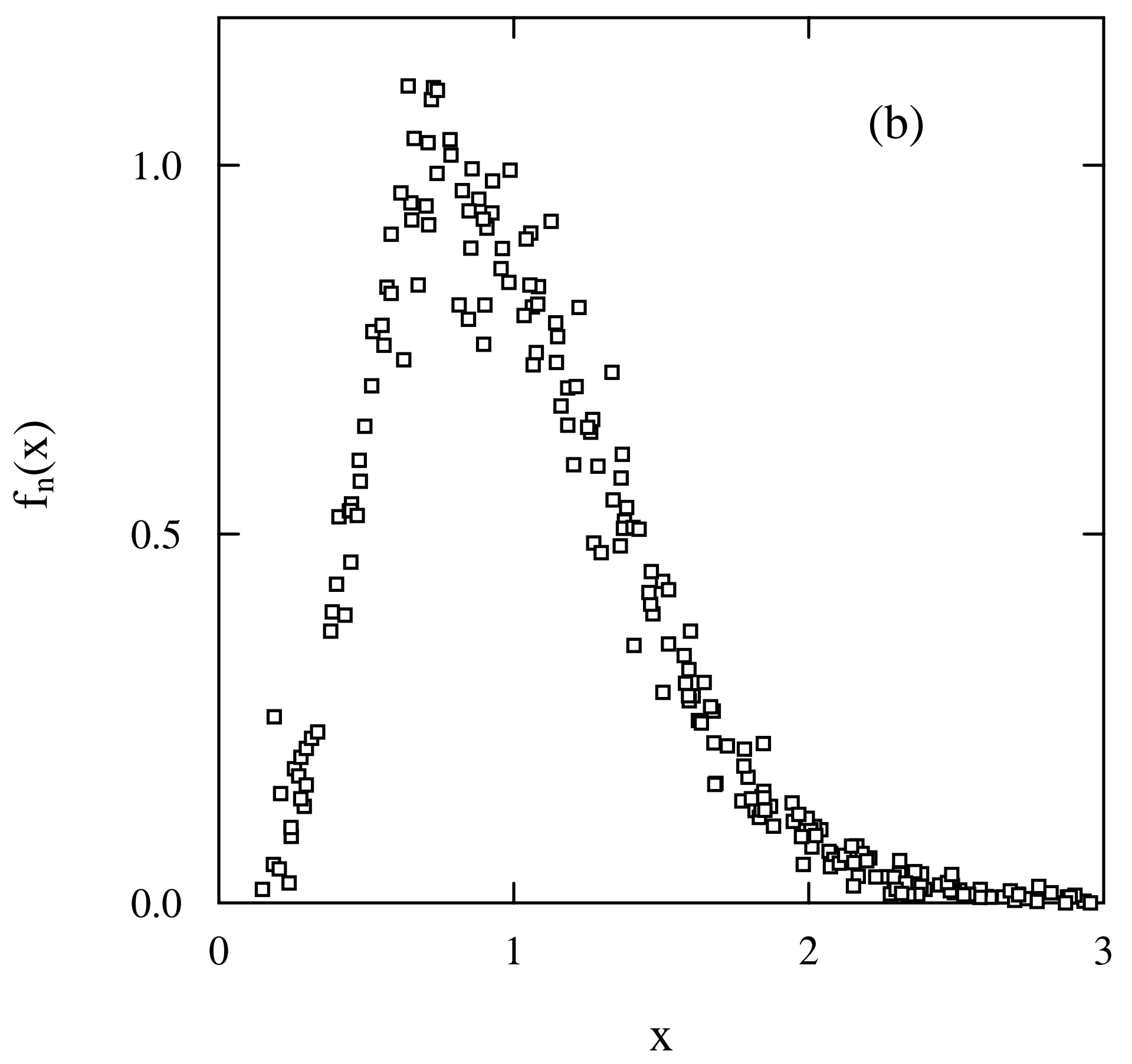


Szeto et al Fig. 2c

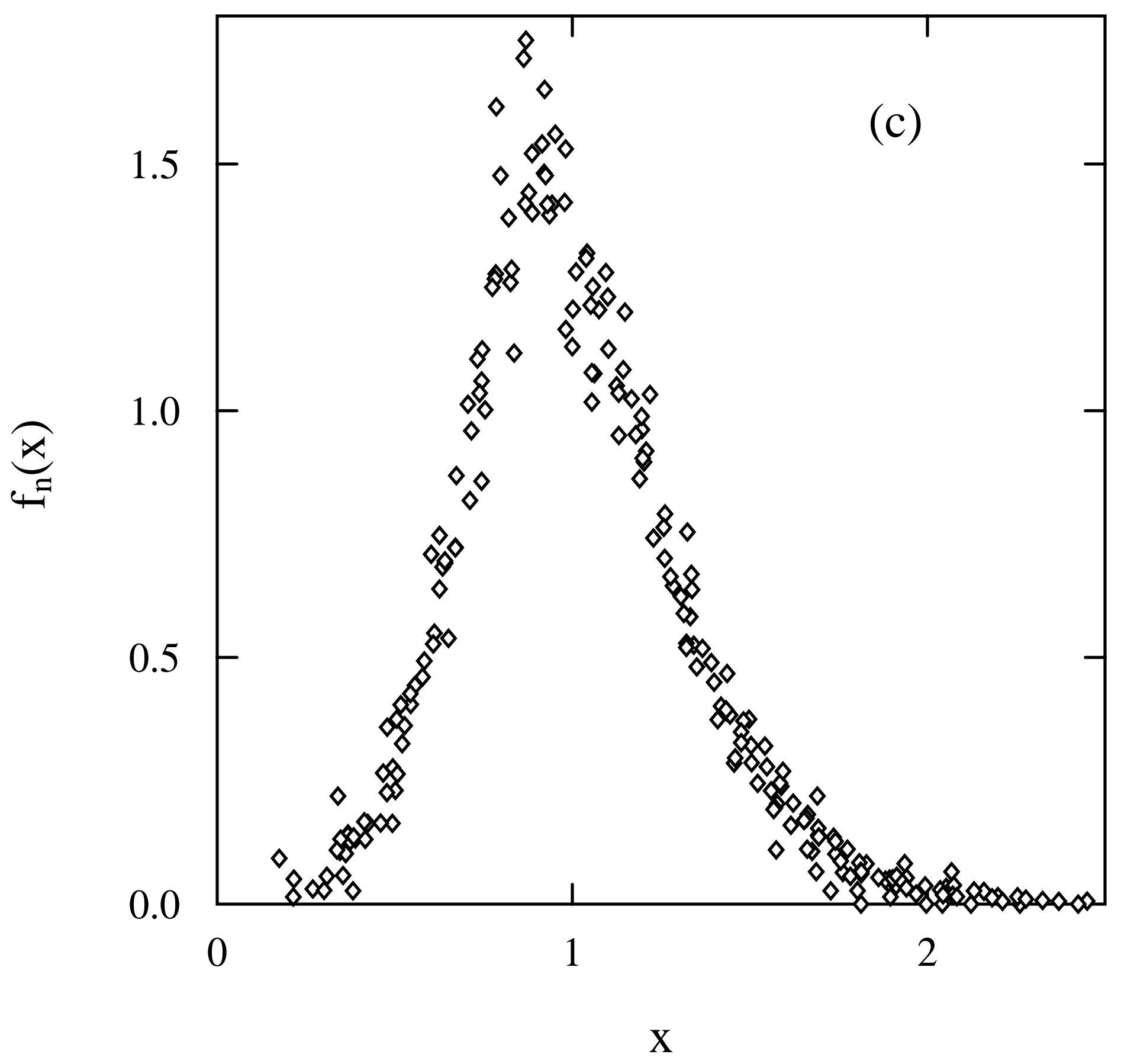


Szeto et al Fig. 3a

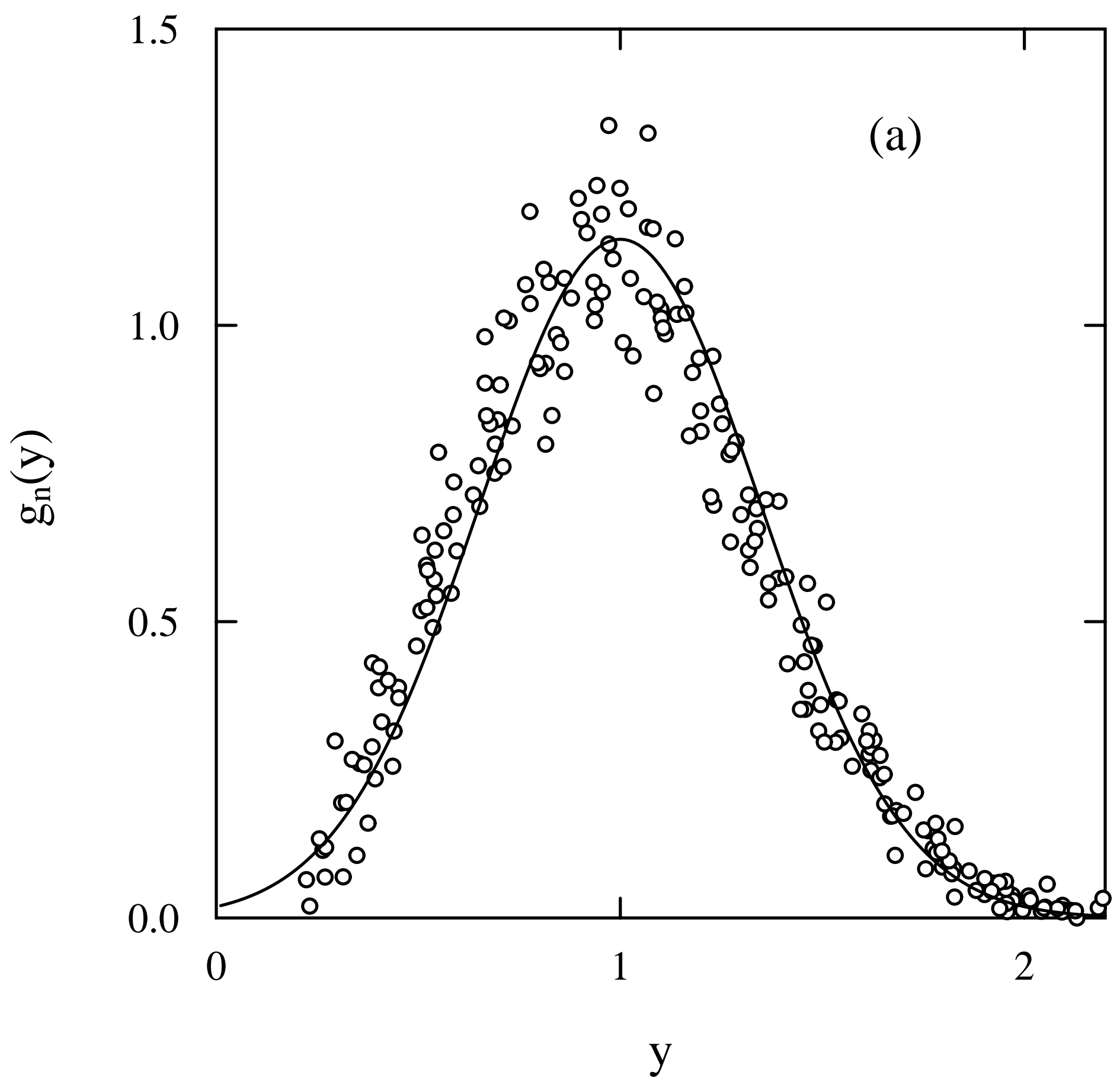


Szeto et al Fig. 3b

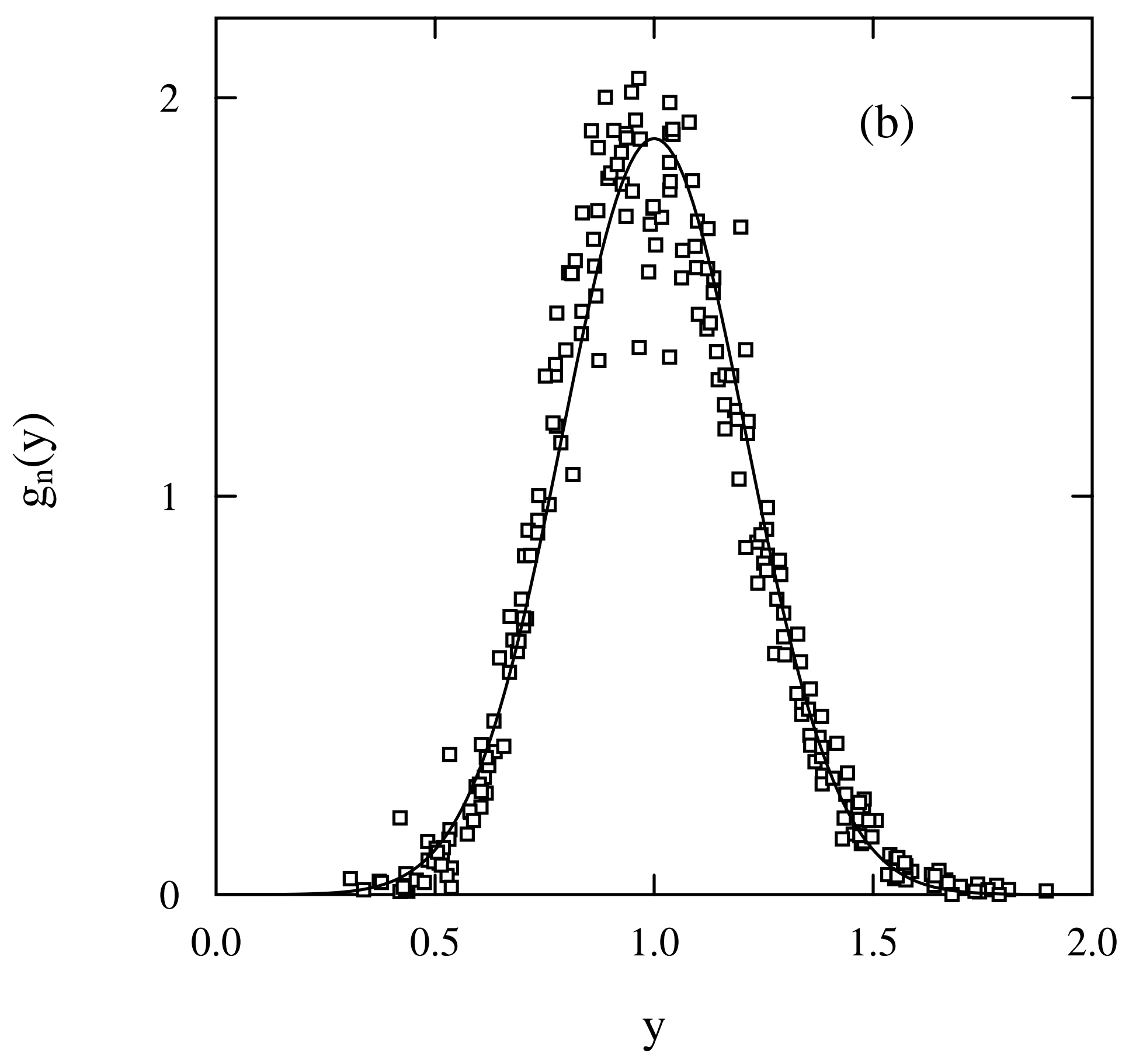


Szeto et al Fig. 3c

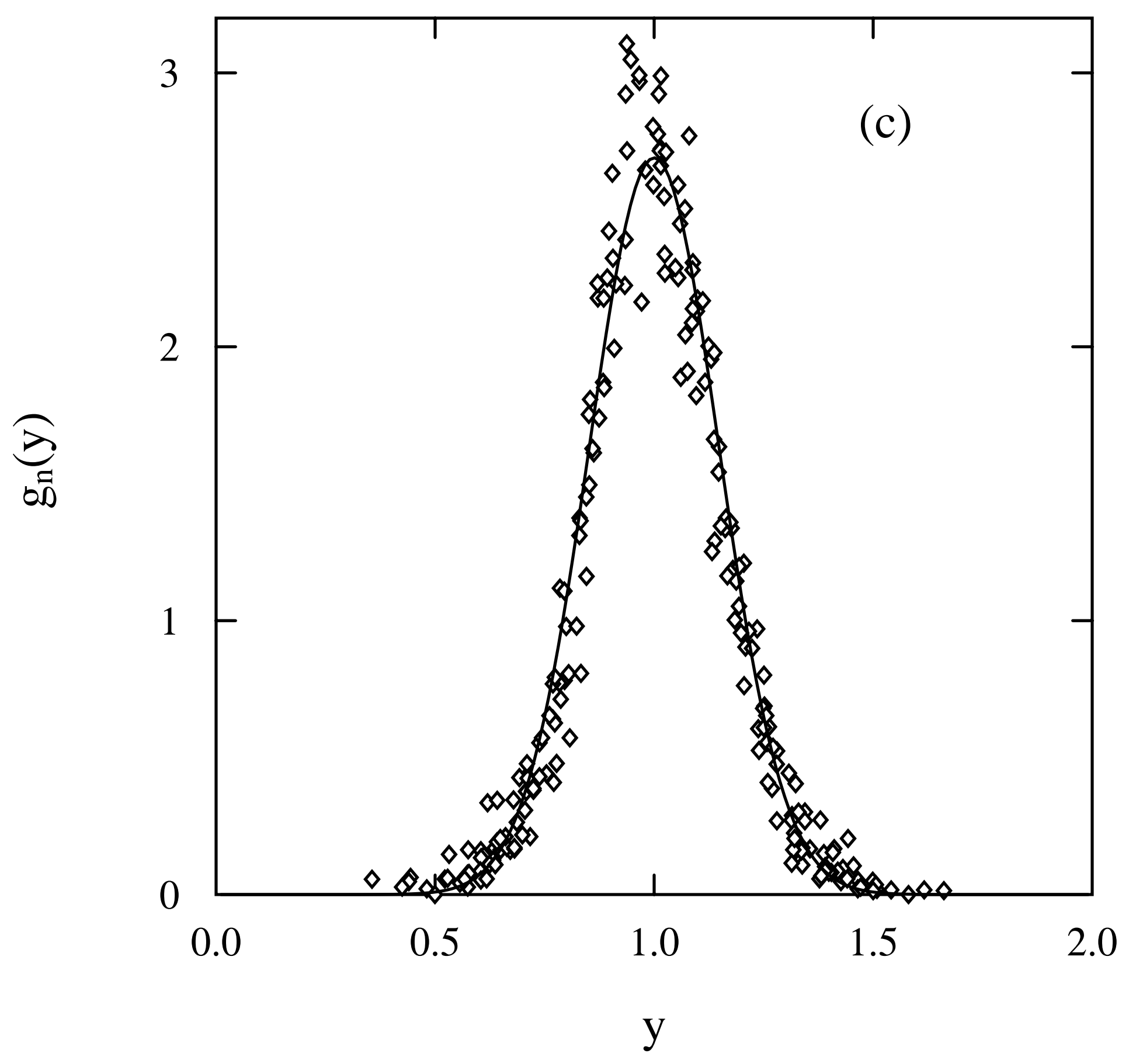


Szeto et al Fig. 4

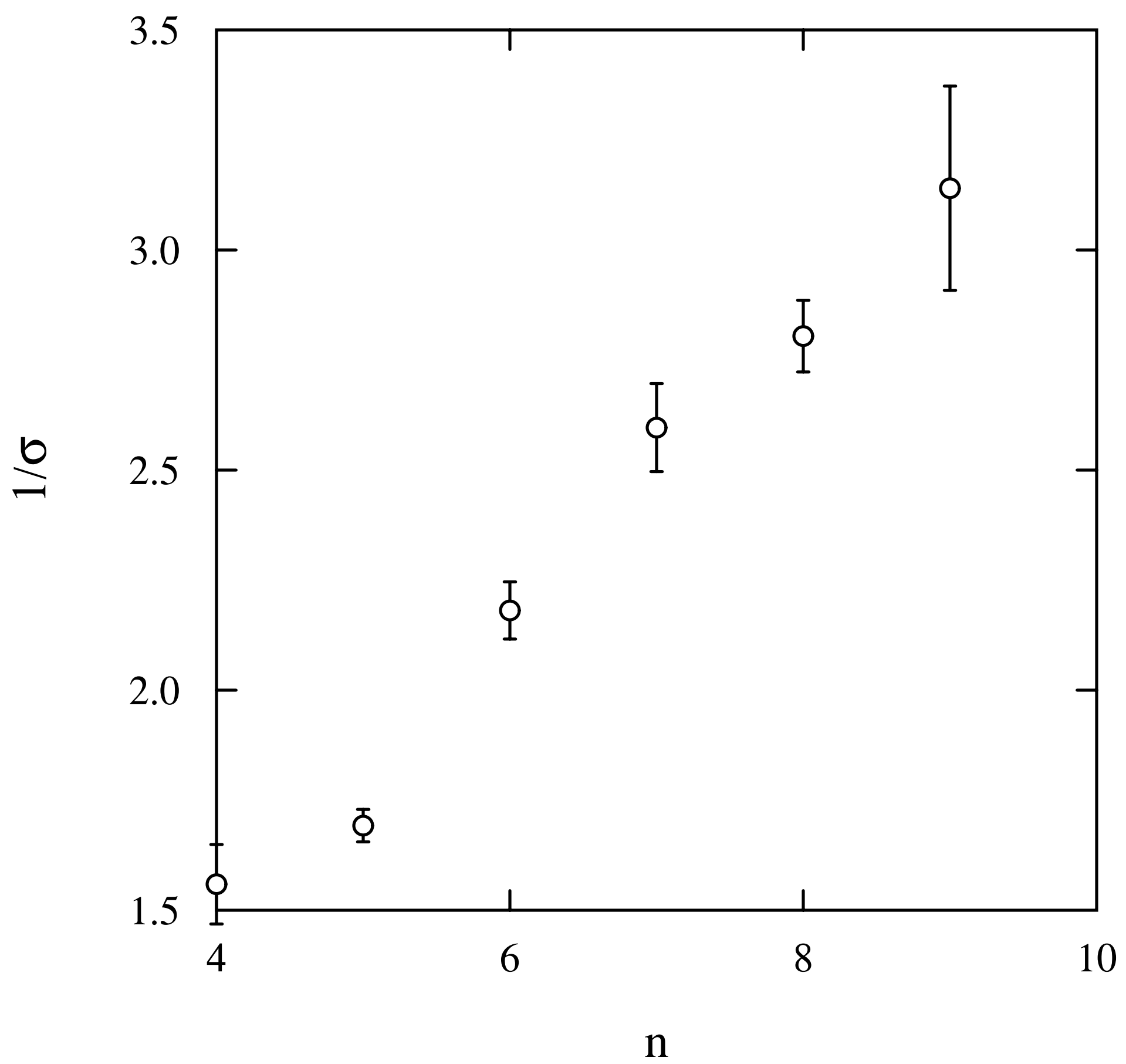

